\title{
LA ACCIÓN SOCIAL EN LA UNIÓN EUROPEA: EVOLUCIÓN HISTÓRICA
}

\section{SILVIA QUESADA DÍEZ}

Licenciada en Ciencias Empresariales y Derecho. Universidad Pontificia de Comillas. ICADE.

\section{RESUMEN}

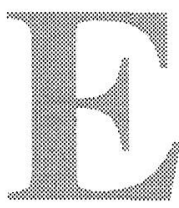

n el presente artículo se va a desarrollar brevemente cuál ha sido la evolución de la Política de Acción Social Europea en los últimos tiempos. Son muchos los esfuerzos que desde la Unión Europea se están llevando a cabo para desarrollar y consolidar una Política Social que busque el mayor beneficio para todos los ciudadanos. Algunos de los principales objetivos han sido, entre otros, mejorar la situación del empleo, el desarrollo de recursos humanos, fomentar la solidaridad y la integración, la lucha contra la pobreza y la exclusión, fomento de la igualdad de oportunidades entre hombres y mujeres en una sociedad europea en curso de transformación, reforzamiento del diálogo social y el mercado único y la libre circulación de las personas.

\section{SUMMARY}

In this article the most important points and the evolution of the Social Policy in Europe it is going to be explained and summarized. A lot of effort is being developing in order to create and consolidate the social profits for the European people. The European Social Policy have specific and important objectives and some of these essential objectives are: to improve the employment situation, to fight against the poverty, to develop the equal opportunities between both, men and women, and to get a global trade in Europe where products and people are able to circulate without any kind of barriers. 


\section{ANTECEDENTES}

Quienes piensan que la Unión Europea es algo distante de la vida del ciudadano de a pie deberían examinar más de cerca su Política Social y los esfuerzos que realiza en materia social. El modelo europeo de acción social ha hecho mucho para mantener la armonía social y la paz durante los cincuenta últimos años. Su mantenimiento continúa siendo una de las prioridades de la Unión y de sus Estados miembros. Todo ello a pesar de las presiones para reducir los gastos sociales que impone, en parte, la competencia mundial y que se han intensificado en un momento en que el aumento del desempleo y el envejecimiento de la población lastran cada vez más el presupuesto de la Unión.

El desarrollo de una política de acción social a escala comunitaria no figuró entre las grandes prioridades de los seis Estados miembros durante los doce primeros años de la Comunidad Europea. El único sector de actividad real era la coordinación de los regímenes de seguridad social para los trabajadores emigrantes que ejercían el derecho de libre circulación que les confería el Tratado.

Las disposiciones de política social, que ya figuraban en el Tratado constitutivo de la Comunidad Europea del Carbón y del Acero (1951), se vieron confirmadas más tarde en el Tratado constitutivo de la CEE con la inclusión de la mejora de las condiciones de vida y de trabajo entre los objetivos de la integración europea. Para ello se contaba, sobre todo, con la contribución del Fondo Social Europeo y con la coordinación y cooperación entre los Estados miembros. Ya antes de 1970, y como parte de la realización de la libre circulación de los trabajadores, uno de los objetivos fundamentales del mercado interior, se creó un sistema para garantizar las prestaciones de seguridad social a los trabajadores que ejercieran su actividad laboral en otro Estado miembro.

Para poder comprender mejor todo el esfuerzo desarrollado por la Unión Europea en materia de Acción Social, creemos necesario que el lector conozca una breve historia sobre lo que ha ido sucediendo en materia social durante las últimas décadas.

\section{PRIMEROS TIEMPOS (1960): FONDO SOCIAL}

«Para mejorar las posibilidades de empleo de los trabajadores en el mercado interior y contribuir así a la elevación del nivel de vida, se crea, en el marco de las disposiciones siguientes, un Fondo Social Europeo destinado a fomentar, dentro de la Comunidad, las oportunidades de empleo y la movilidad geográfica y profesional de los trabaja- 
dores, así como a facilitar su adaptación a las transformaciones industriales y a los cambios de los sistemas de producción, especialmente mediante la formación y la reconversión profesionales», según se explica en el art. 123 TCE. La administración del Fondo corresponde a la Comisión.

El Fondo fue creado en el Tratado de Roma y empezó sirviendo principalmente para financiar medidas de reinstalación y de reconversión profesional y beneficiando en mayor medida a los Estados miembros más «ricos» que poseían un mayor experiencia en la presentación de solicitudes. A lo largo de los años ha sido objeto de varias revisiones y modificaciones desde su instauración hasta nuestros días.

El FSE se concentra, esencialmente, en ayudas al empleo y en formación profesional continua de los trabajadores a través de acciones de formación, tal y como establecen los art. 125 y 130 E del TCE. Así, los principales objetivos del FSE son conseguir:

- La integración a la vida laboral de personas desempleadas durante un largo período.

- Integración a la vida laboral de los jóvenes en busca de empleo.

- Integración de personas expuestas a la exclusión del mercado de trabajo.

- Promoción de la igualdad de oportunidades entre ambos sexos en el mercado laboral.

- Anticiparse al desempleo adaptando a los trabajadores al cambio laboral.

- Estabilidad y crecimiento del empleo, impulsando la creación de puestos de trabajo.

- Fortalecimiento de los sistemas de educación y formación.

Entre 1994 y 1999 el FSE transferirá 47.000 millones de ecus del presupuesto de la Unión Europea para cofinanciar acciones emprendidas por los Estados miembros.

\section{III.PRIMER PROGRAMA (1974)}

Hasta la adopción del primer Programa de Acción Social en 1974, que marcó el verdadero comienzo de la política social comunitaria, ésta estuvo limitada a las actividades del Fondo Social Europeo. A partir de mediados de los años setenta, la política de acción social se centró en programas de objetivos específicos como, por ejemplo, los dedicados a la seguridad y salud en el lugar de trabajo, al fomento de la igualdad de oportunidades para las mujeres y a la inserción en la vida laboral de los grupos desfavorecidos. Sólo pocas de estas actividades podían apoyarse en un fundamento jurídico específico y los programas 
de Política de Acción Social se basaban en las normas generales de competencia, así y todo, desde 1974 se aceptó básicamente la competencia de la CE para promulgar disposiciones en materia de Política Social.

\section{ACTA ÚNICA (1987)}

El Acta Única Europea fue firmada en 1986 y entró en vigor el I de julio de 1987. Apenas supuso una ampliación de las competencias comunitarias en el terreno de la Política de Acción Social: el nuevo artículo $118 \mathrm{~A}$ del Tratado CEE sólo preveía las medidas para salvaguardar la salud y la seguridad en el lugar de trabajo que pudieran ser adoptadas por el Consejo por mayoría cualificada y en cooperación con el Parlamento Europeo.

El Acta Única corrobora en más de un aspecto la evolución durante los años anteriores en materia de desarrollo de políticas comunes y confirma el objetivo de completar el mercado único europeo en 1992 según el calendario fijado en el Libro blanco de 1986. El Tratado constitutivo de la CEE queda enriquecido con la aportación que hace el Acta Única Europea de nuevos artículos que dan un nuevo impulso a la Política de Acción Social, sugieren la importancia de estimular el diálogo entre los interlocutores sociales y hacen hincapié en la necesidad de la cohesión económica y social de los doce Estados miembros.

\section{LA CARTA SOCIAL (1989)}

La Carta Comunitaria de los Derechos Sociales Fundamentales de los Trabajadores fue adoptada por el Consejo Europeo en diciembre de 1989, pese al voto contrario del Reino Unido. Esta Carta pretende contribuir a la realización de la dimensión social del mercado interior. Sin embargo, a raíz de los debates a que ésta fue sometida en el Consejo y a la postura negativa del Reino Unido, el ambicioso objetivo inicial de una "Carta que estableciera unos derechos fundamentales vinculantes y reclamables" quedó convertido en una mera declaración de intenciones políticas de carácter no vinculante sobre una serie de derechos sociales fundamentales.

Los derechos fundamentales que se contienen en ella, como por ejemplo la formación profesional y la libertad de asociación, el derecho de los trabajadores y a la información, etc., no pueden reclamarse, sino que sirven de directrices para la política en los Estados miembros de la Unión Europea, salvo en Gran Bretaña. La Carta Social recoge doce principios: 
1. El derecho a elegir el Estado miembro en que se desea trabajar.

2. El derecho a una remuneración justa.

3. El derecho a mejores condiciones de vida y trabajo.

4. El derecho a la protección social con arreglo a la realidad de cada Estado miembro.

5. La libertad de asociación sindical y el derecho a la negociación colectiva.

6. El derecho a la formación profesional.

7. El derecho a la igualdad de trato de hombres y mujeres.

8. El derecho de información, consulta y colaboración de los trabajadores.

9. El derecho a la protección de la salud y a la seguridad en el puesto de trabajo.

10. La protección de la infancia y la juventud.

11. El derecho de las personas mayores a disfrutar de un nivel de vida adecuado.

12. La promoción de la integración social y profesional de los minusválidos.

\section{SEGUNDO PROGRAMA DE ACCIÓN SOCIAL (1989)}

A pesar de lo acontecido con la Carta Social, y a fin de poder llevar adelante la política social, la Comisión presentó aún en diciembre de 1989 un programa de acción para la aplicación de la Carta a través de cuarenta y siete medidas concretas. Las iniciativas propuestas, que deben contar con la aprobación del Consejo, se han llevado a la práctica en gran parte. Entre ellas, figuran, por ejemplo, directivas sobre los despidos colectivos, sobre la prueba de la relación laboral, sobre la protección de la maternidad, sobre la protección del trabajo juvenil y sobre la jornada laboral. La realización de las medidas restantes se llevará a cabo probablemente, en parte, sin el Reino Unido, en el marco del Protocolo Social.

\section{MAASTRICHT Y EL PROTOCOLO SOBRE LA POLÍTICA SOCIAL (1991)}

En el transcurso de las negociaciones para el Tratado de la Unión Europea el Gobierno británico sostuvo la no ampliación de las competencias comunitarias. Por ello, se temió que la Política Social fuera a 
convertirse en el obstáculo para la llegada a un acuerdo. El Consejo Europeo de Maastricht (diciembre de 1991) acabó encontrando un compromiso consistente en dejar intactas las disposiciones del Tratado CEE en materia de Política Social y en añadirle un «Protocolo sobre la política social». En dicho Protocolo, firmado por los doce Estados miembros y anexo al Tratado de Unión Europea, se declara la voluntad de once Estados miembros de lograr avances significativos en materia de política social.

Mediante este Protocolo se facultaba a los demás Estados miembros a recurrir a los órganos, procedimientos y mecanismos de los Tratados para llevar adelante una política social común de mayor alcance sin el Reino Unido. Lo expuesto anteriormente llevó consigo que la aplicación del Protocolo Social diera lugar, por primera vez en la historia de la Comunidad, a un acto de legislación comunitaria derivada destinado solamente a una parte de los Estados miembros, pudiendo crear con ello una posible ventaja competitiva para el Reino Unido. Sin embargo, en mayo de 1997, el Reino Unido anunció su intención de renunciar a su cláusula de excepción en materia social.

Los objetivos de la Política de Acción Social son el fomento del empleo, la mejora de las condiciones de vida y de trabajo, una protección social adecuada, el diálogo social, el desarrollo de los recursos humanos para conseguir un empleo elevado y duradero y la lucha contra las exclusiones.

Por último, sitúa explícitamente fuera del ámbito de las competencias comunitarias las remuneraciones, el derecho de asociación y sindicación, el derecho de huelga y el derecho de cierre patronal.

\section{LIBRO VERDE (1993)}

En noviembre de 1993, la Comisión publicó el Libro Verde sobre el futuro de la Política Social europea en el marco de un extenso proceso de consulta. Parecía claramente necesario que los ciudadanos europeos debían sentir que la Unión y la Europa social les afectaba más que en el pasado. Los mensajes clave resultantes del proceso de consulta y recogidos en el Libro verde eran que existe un modelo social europeo claro, basado en la democracia y los derechos individuales, la libertad de negociación colectiva, la economía de mercado, la necesidad de igualdad de oportunidades para todos y la importancia de la protección social y de la solidaridad.

Europa atravesaba un período de cambios profundos a nivel social y económico, como muestra la mundialización creciente, la difusión de nuevas tecnologías, los nuevos métodos de organización del traba- 
jo, la evolución de las estructuras de la población en Europa y el aumento del coste de la asistencia sanitaria de las pensiones. El Libro Verde concluye que el desempleo en Europa es un problema estructural y no cíclico y debe tratarse como tal. No basta con que el PIB aumente; es necesario que el empleo se desarrolle paralelamente.

\section{Resumen de las principales cuestiones}

1. En materia de mercado de trabajo:

- la oferta de un empleo o de una formación a todos aquellos que buscan trabajo,

- un nuevo equilibrio entre los gastos dedicados, por una parte, a las medidas activas y, por otra, al mantenimiento de los ingresos.

2. En materia de protección social y de exclusión:

- establecimiento de un nivel mínimo de ingresos,

- plan de integración para todos los excluidos.

3. En materia de igualdad de oportunidades:

- objetivos (trabajo compartido, reparto de los ingresos, toma de decisiones) en favor de grupos específicos tales como las mujeres,

- medidas que permitan combinar las responsabilidades familiares y el trabajo,

- en cuanto a los jóvenes, la «garantía» del empleo o de una formación útil,

- medidas que permitan fomentar el fin de la segregación vertical y horizontal del mercado de trabajo.

4. En materia de formación:

- objetivos en materia de producción de cualificaciones en diferentes niveles, de forma que se movilice a los diferentes actores;

- supresión de los obstáculos jurídicos todavía existentes a la libre circulación de los trabajadores (reagrupación familiar, igualdad de trato, derecho de residencia, etc.);

- desarrollo de la red EURES a fin de proporcionar asesoramiento e información sobre las ofertas de empleo y las condiciones de vida y de trabajo a escala comunitaria;

- igualdad de trato para todos los ciudadanos de la UE que residan en un Estado miembro, en particular en lo que se refiere a las ventajas sociales y fiscales (estudiantes, jubilados, etc.);

- garantía de una mejor protección social de los trabajadores que se trasladan a otro país comunitario, especialmente facilitando la adquisición, la conservación y la transferencia de los derechos de jubilación;

- ampliación de la población cubierta por la coordinación de los regímenes de seguridad social, simplificación de las normas, mo- 
dernización de los procedimientos y mejor información del público en general.

\section{LIBRO BLANCO (1994)}

En julio de 1994, la Comisión publicó el Libro Blanco sobre la Política de Acción Social europea, que contenía sus propuestas sobre el camino a seguir. El Libro Blanco aboga por una nueva amalgama de Políticas Sociales y Económicas y hace hincapié en el hecho de que la competitividad y el progreso social pueden ir unidos. Indica que Europa tiene sobre todo necesidad de una mano de obra adaptable, cualificada y motivada, que solamente una política social puede generar. Los grandes temas del Libro Blanco son: el empleo, las modalidades de desarrollo del fundamento jurídico y de la necesidad vital de una sociedad que exija la colaboración activa de todos.

El Libro Blanco es el resultado del procedimiento de consulta iniciado por el Libro Verde sobre la Política Social europea que ha implicado a las instituciones de la Unión, a los Estados miembros, a los empresarios, a los sindicatos, a las instituciones públicas y a los particulares. Se esfuerza por determinar las principales líneas de acción de la Unión para los próximos años y las principales propuestas son relativas al empleo y a la formación.

Este Libro pretende consolidar y desarrollar todo lo que se ha ido realizando anteriormente, en particular lo que respecta al derecho laboral, la salud y seguridad, la libre circulación y la igualdad de trato a mujeres y hombres. Se hacen nuevas propuestas en los sectores de la protección social, la igualdad de oportunidades para todos y la salud pública.

\subsection{Resumen de las principales cuestiones.}

1. Empleos: la primera prioridad. La Comisión propone en el ámbito del empleo, entre otras muchas:

- reforzar su cooperación existente con las autoridades de los Estados miembros;

- desarrollar la cooperación en la Unión entre todos los directores generales con responsabilidades en materia de empleo, incluidos los ministerios de Empleo, Protección social, Educación y Formación;

Las PYMES son creadoras de empleo, y la Comisión, en el marco de su programa integrado en favor de las PYMES concede una atención particular al estímulo de las mejoras del entorno administrativo y legal de las PYMES. 


\section{Invertir en una mano de obra de primer orden.}

La inversión en educación y formación es una de las condiciones esenciales de la competitividad de la Unión y de la cohesión de nuestras sociedades. Las principales propuestas vinculadas al plan global de acción para el empleo, concentrándose en las prioridades fijadas en el Libro Blanco sobre crecimiento, competitividad y empleo y con la voluntad, en el marco de las estructuras nacionales, fueron, entre otras:

- garantizar en toda la Unión que ningún joven esté en el paro antes de la edad de 18 años;

- fijar objetivos escalonados hasta el año 2000 para la eliminación del analfabetismo y de la falta de cualificaciones de base entre los jóvenes que dejan la escuela;

- mejorar el estatuto de la formación y de la enseñanza profesionales iniciales y fomentar, gracias a una experiencia profesional conveniente, el desarrollo del espíritu de empresa en los jóvenes, así como sus capacidades de explotar las nuevas tecnologías;

Los Fondos Estructurales, gracias a un presupuesto de más de 141.000 millones de ecus para el período 1994-1999, han contribuido de manera importante a la inversión en recursos humanos, a la lucha contra el desempleo y al buen funcionamiento del mercado del empleo. En cuanto al Fondo Social Europeo, su dotación ha sido de más de 40.000 millones de ecus para el mismo período. El FSE en el conjunto de la Unión tiene por tarea luchar contra el desempleo de larga duración y la exclusión del mercado del empleo, intentar dar a todos los jóvenes las cualificaciones necesarias y la posibilidad de trabajar, promover la igualdad de oportunidades y, en el marco del objetivo n. ${ }^{\circ} 4$, ayudar a la mano de obra a adaptarse a los cambios industriales.

3. Promover mejores normas de empleo para una Europa competitiva

Los Estados miembros siguen divididos sobre si es oportuno adoptar nuevas medidas legislativas sobre las normas de empleo a nivel europeo. Se ha propuesto una serie de sectores para la acción legislativa de la Unión, como la protección contra los despidos individuales, la protección de la vida privada de los trabajadores, en particular, en cuanto al tratamiento de los datos personales, incluidas su recogida y su difusión, etc.

4. La seguridad social.

Se realizaría un examen y una reestructuración técnica de gran envergadura de las reglas de coordinación de las disposiciones de la seguridad social para adaptarlas a los cambios y necesidades de los particulares y para simplificarlas. La principal cuestión ha sido y es, la necesidad de mantener una protección adecuada de las per- 
sonas que circulan de un país a otro por lo que respecta a los regímenes profesionales o a cualquier otro régimen complementario de seguridad social que se añada o que sustituya a lo establecido por ley.

5. Igualdad de oportunidades entre los hombres y las mujeres

Suprimir la segregación que existe en el mercado del empleo y valorar el trabajo de las mujeres. Así, la Comisión:

- introducirá códigos de buena práctica en materia de igualdad de remuneraciones por un trabajo de igual valor, de formación y de eliminación de la segregación vertical;

- adoptará medidas tendentes a fomentar la adquisición de competencias y el perfeccionamiento profesional de las mujeres;

- presentará propuestas con vistas a la eliminación de políticas discriminatorias en materia de fiscalidad y de protección social y con vistas a la individualización de los derechos;

- emprenderá una evaluación económica de la creación de empleos y del potencial de reanudación de las infraestructuras y de los servicios para niños y personas dependientes;

- continuará sus trabajos de investigación, información y formación sobre una mayor participación de las mujeres en las tomas de decisión en los todos los sectores.

6. Acción en el sector de la salud pública.

La Comisión ha aprobado propuestas de decisión para tres de estos programas, que son los relativos al cáncer, la promoción, la información, la educación y la formación en materia de salud y la prevención de las toxicomanías.

La Comisión propone prioritariamente otros programas sobre el SIDA y otras enfermedades transmisibles, así como sobre los datos sanitarios y los indicadores de salud, incluida la vigilancia de las enfermedades. Se prevén otros programas en sectores como los accidentes y las heridas, las enfermedades vinculadas a la contaminación y las enfermedades raras.

7. Los sindicatos, las organizaciones patronales y las organizaciones de voluntarios como socios del cambio.

8. Cooperación internacional: el papel de la política social europea.

La Comisión tiene la intención de:

- dar un carácter sistemático a los intercambios y a los contactos con otros países industrializados;

- proseguir su larga tradición de cooperación con la organización internacional del trabajo. La Comisión, hasta la ampliación de la UE en 1995, seguirá contribuyendo activamente a la cooperación en materia de política social en el marco del acuerdo del Espacio Económico Europeo; 
- ayudar a los países de Europa central y oriental a encontrar respuestas a sus problemas (consecuencias sociales de las reestructuraciones industriales, aplicación de las políticas de empleo, lucha contra la pobreza y la exclusión social, instauración de regímenes de seguridad social, etc.) y organizar la cooperación, con la ayuda y el apoyo de los Estados miembros.

\section{TERCER PROGRAMA DE ACCIÓN SOCIAL (1995-...)}

Este programa es la tercera y última fase de un proceso que comenzó en 1993 con la publicación del Libro Verde sobre el futuro de la Política Social europea y que prosiguió en 1994 con el Libro Blanco. Los puntos fundamentales del programa son:

- Utilización más eficaz del FSE para promover el empleo.

- Fomento de la formación general y profesional.

- Facilitar la libre circulación dentro de la Unión Europea.

- Creación de una estructura de normas mínimas en todos los ámbitos de la política social.

- Regulación de la compatibilidad de la vida familiar y profesional.

- Aplicación del principio de igualdad de oportunidades en todos los ámbitos.

- Asistencia para los pobres, minusválidos y ancianos.

- Actuación conjunta para luchar contra el racismo y la xenofobia en la sociedad.

- Profundizar en el diálogo social.

- Intensificar la colaboración con organizaciones no gubernamentales.

- Analizar las repercusiones sociales de la sociedad de la información.

En un informe del Parlamento Europeo sobre este programa de medidas, los diputados exigen una mayor perspectiva social de Europa, que debe expresarse sobre todo a través de los interlocutores sociales. Los cambios estructurales que comporta el mercado interior deben afrontarse a través del diálogo entre empresarios y trabajadores. De ahí debe surgir la legislación europea, como en el caso del permiso parental o del debate sobre el trabajo a tiempo parcial, para que puedan protegerse con plena eficacia los derechos de los trabajadores.

El gráfico que se muestra a continuación expresa el reparto de los fondos del FSE durante el año 1997. 


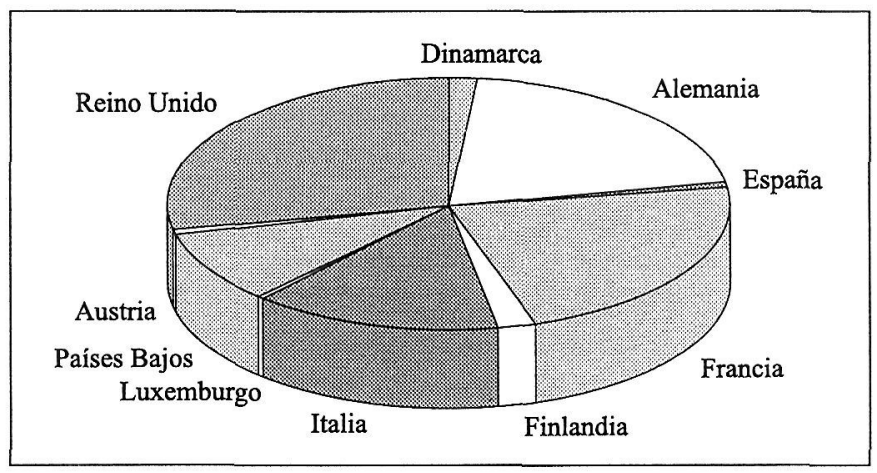

\section{CONCLUSIÓN}

Todo lo expuesto en el presente artículo ha sido, principalmente, la evolución histórica y los objetivos y metas a alcanzar por la Política de Acción Social Europea en los últimos años. Habrá que constatar que en los años venideros todas estas iniciativas y proposiciones han seguido dado fruto incrementando y mejorando la calidad de vida de todo ciudadano europeo.

Creemos que los ciudadanos de la Unión Europea deben poseer un mayor conocimiento de todos los esfuerzos que desde Europa se están llevando a cabo en materia de Política Social. Es algo que nos concierne a todos y que se está desarrollando para nuestro beneficio. La Política de Acción Social es fundamental en toda sociedad que busque el beneficio de sus ciudadanos y este es un objetivo primordial de la Unión Europea.

\section{BIBLIOGRAFÍA}

ALONSO GARCÍA, R. (1996): Tratado de la Unión Europea. Civitas. $4^{\text {a }}$ edición. Colegio de Economistas de Madrid. (1996) «Política de Empleo en Europa».

WERNER WEIDENSELD, W. (1997): Europa de la A a la Z. Guía de la integración europea. Oficina de Publicaciones Oficiales de las Comunidades Europeas. Luxemburgo.

Economic Commission for Europe United Nation «Economic Survey of Europe 1996-1997». Ginebra. NN.UU. 1997. 